\title{
Health status in patients with coexistent COPD and heart failure: a validation and comparison between the Clinical COPD Questionnaire and the Minnesota Living with Heart Failure Questionnaire
}

This article was published in the following Dove Press journal:

International Journal of COPD

22 September 2014

Number of times this article has been viewed

\author{
Farida F Berkhof' \\ Leola Metzemaekers' \\ Steven M Uil' \\ Huib AM Kerstjens ${ }^{2}$ \\ Jan WK van den Berg' \\ 'Department of Pulmonary Diseases, \\ Isala Hospital, Zwolle, ${ }^{2}$ Department \\ of Pulmonary Diseases, University \\ Medical Center Groningen, University \\ of Groningen, Groningen Research \\ Institute for Asthma and COPD, \\ Groningen, the Netherlands
}

Background: Chronic obstructive pulmonary disease (COPD) and heart failure (HF) are both common diseases that coexist frequently. Patients with both diseases have worse stable state health status when compared with patients with one of these diseases. In many outpatient clinics, health status is monitored routinely in COPD patients using the Clinical COPD Questionnaire (CCQ) and in HF patients with the Minnesota Living with Heart Failure Questionnaire (MLHF-Q) This study validated and compared which questionnaire, ie, the CCQ or the MLHF-Q, is suited best for patients with coexistent COPD and HF.

Methods: Patients with both COPD and HF and aged $\geq 40$ years were included. Construct validity, internal consistency, test-retest reliability, and agreement were determined. The ShortForm 36 was used as the external criterion. All questionnaires were completed at baseline. The CCQ and MLHF-Q were repeated after 2 weeks, together with a global rating of change.

Results: Fifty-eight patients were included, of whom 50 completed the study. Construct validity was acceptable. Internal consistency was adequate for CCQ and MLHF-Q total and domain scores, with a Cronbach's alpha $\geq 0.70$. Reliability was adequate for MLHF-Q and CCQ total and domain scores, and intraclass correlation coefficients were $0.70-0.90$, except for the CCQ symptom score (intraclass correlation coefficient 0.42 ). The standard error of measurement on the group level was smaller than the minimal clinical important difference for both questionnaires. However, the standard error of measurement on the individual level was larger than the minimal clinical important difference. Agreement was acceptable on the group level and limited on the individual level.

Conclusion: CCQ and MLHF-Q were both valid and reliable questionnaires for assessment of health status in patients with coexistent COPD and HF on the group level, and hence for research. However, in clinical practice, on the individual level, the characteristics of both questionnaires were not as good. There is room for a questionnaire with good evaluative properties on the individual level, preferably tested in a setting of patients with COPD or HF, or both.

Keywords: Clinical COPD Questionnaire, Minnesota Living with Heart Failure Questionnaire, chronic obstructive pulmonary disease, heart failure

\section{Introduction}

Chronic obstructive pulmonary disease (COPD) is a major worldwide cause of morbidity and mortality, and its prevalence is predicted to increase over the following decades. ${ }^{1}$ COPD is an inflammatory disease with extrapulmonary manifestations, 
including an increased prevalence of cardiovascular disease in comparison with the general population. ${ }^{2}$ Nine percent to $41 \%$ of COPD patients have heart failure (HF). ${ }^{3}$ Both systemic conditions have certain pathophysiologic characteristics in common, ie, the shared risk factor of smoking leading to low-grade systemic inflammation, which in turn accelerates progression of atherosclerosis in both diseases. ${ }^{4}$ COPD and HF also have overlapping clinical manifestations, like dyspnea, fatigue, and exercise intolerance. ${ }^{5,6}$ Managing patients with combined COPD and HF is a challenge because it is difficult to determine whether signs and symptoms are caused by COPD, HF, or both. This can lead to delays in adequate treatment, to more severe exacerbations, and to more frequent hospital admissions. ${ }^{7}$ Patients hospitalized with an exacerbation of COPD and HF have more hospital days, more readmissions, and higher mortality compared with COPD patients without HF. ${ }^{8}$ COPD patients with combined disease also have more frequent exacerbations, leading to worse health status. ${ }^{9}$ Further, patients with coexistent COPD and HF have a worse health status in the stable state than patients with COPD only. ${ }^{10}$ Improving health status is a treatment goal in the follow-up of COPD patients. ${ }^{11}$ Thus, it is important to monitor health status routinely in the outpatient clinic. Unfortunately, many patients have more than one chronic condition. This makes it difficult to use a single disease-specific health status questionnaire. In addition, measuring health status using several questionnaires may be troublesome for the patient. For COPD patients, the most frequently used questionnaire in the Netherlands is the Clinical COPD Questionnaire (CCQ). ${ }^{12}$ For HF patients, the Minnesota Living with Heart Failure Questionnaire (MLHFQ) is most frequently used. ${ }^{13}$ Both health status questionnaires, ie, the CCQ and the MLHF-Q, are disease-specific questionnaires. Therefore, patients with coexistent COPD and HF theoretically have to complete both questionnaires, which is time-consuming and not practical in the outpatient clinic. One questionnaire to assess health status for patients with both diseases, ie, COPD and HF, would be ideal. Unfortunately, no health status questionnaire has been constructed for patients with coexistent COPD and HF. Therefore, the aim of this study was to compare which existing questionnaire, the CCQ or the MLHF-Q, is suited best for patients with coexistent COPD and HF.

\section{Materials and methods}

\section{Study design}

This single-center, prospective, validation study was carried out at Isala Hospital, a large teaching hospital in Zwolle, the Netherlands. Stable patients with both HF and COPD were contacted by telephone and invited to participate in the study, and signed their informed consent. Approval was received from the local ethics committee (local number 11.10127).

\section{Patients}

Patients with COPD GOLD (Global Initiative for Chronic Obstructive Lung Disease) stage $\geq 2$ (defined as a post-bronchodilator forced expiratory volume in one second $\left[\mathrm{FEV}_{1}\right]$ $<80 \%$ and a ratio of $\mathrm{FEV}_{1}$ to forced vital capacity of $<70 \%$ ), HF (defined clinically as a syndrome in which patients have typical signs and symptoms of HF and a reduced left ventricular ejection fraction or diastolic dysfunction ${ }^{14}$ ), New York Heart Association functional class $\geq 2$, and aged 40-85 years with a smoking history of $\geq 5$ pack years, and able to provide written informed consent were included. Patients who were not able to complete questionnaires on their own were excluded.

\section{Data collection}

Demographic characteristics, comorbidity (Charlson Comorbidity Index), post-bronchodilator spirometry, pro-B-type natriuretic peptide, and New York Heart Association classification for HF were recorded at baseline. Construct validity, internal consistency, and floor and ceiling effects were determined by administering the CCQ, MLHF-Q, and Short-Form 36 (SF-36) at baseline. To assess reliability and responsiveness, all questionnaires together with the Global Rating of Change (GRC) were administered at week 2 and week 12. Questionnaires were sent to the patients by mail, were completed at home, and were returned by mail.

\section{Questionnaires}

\section{Clinical COPD Questionnaire}

The $\mathrm{CCQ}^{12}$ is a ten-item COPD-specific questionnaire with total, symptom, functional status, and mental status domains. A higher score indicates a worse health status. The minimal clinical important difference (MCID) of the CCQ total score is $0.4 .^{15}$ The CCQ was developed without factor analysis because a discrepancy was found between factor analysis and expert opinion. After deliberation, clinicians and experts in the field of COPD management decided to forego factor analysis and to compose the domains themselves. ${ }^{12}$

\section{Minnesota Living with Heart Failure Questionnaire}

The MLHF-Q is a HF-specific questionnaire consisting of 21 items with a 6-point response scale from 0 to 5 , 
leading to a total score and two domain scores, ie, physical and emotional state. A higher score indicates worse health status. ${ }^{13}$ The MCID was estimated at 4.8. ${ }^{16}$ The MLHF-Q was developed with factor analysis. ${ }^{13}$

\section{Short-Form 36}

The SF-36 is a generic health status questionnaire with eight domains, ie, physical functioning, role physical, bodily pain, general health, vitality, social function, role emotional, and mental health. ${ }^{17-19}$ All scores are transformed to a range from 0 to 100 , with higher scores indicating better health status.

\section{Global Rating of Change}

The GRC was used to assess self-perceived change in health status, "To what extent have your pulmonary and/or cardiac symptoms changed in the past weeks?", on a 15-point scale ( -7 a very great deal worse, 0 no change, +7 a very great deal better). ${ }^{20}$

\section{Statistical analysis}

Data analyses were performed using Statistical Package for the Social Sciences version 20.0 software (IBM Corporation, New York, NY, USA). Baseline characteristics are presented as the mean (standard deviation) unless otherwise stated. In this study, the clinimetrics statistics definitions of the COSMIN (Consensus-based standards for the selection of health status measurement instruments) study were followed. ${ }^{21,22}$ First, one of the authors assessed face validity, the degree to which the items of the CCQ and MLHF-Q indeed look to be an adequate reflection of the construct to be measured, namely "health status in patients with both, COPD and HF". To assess content validity, ${ }^{22}$ the relevance and comprehensiveness of items in the CCQ and MLHF-Q were viewed and the subsequent four questions were answered, ie, "Do all items of the questionnaires refer to relevant aspects of the construct?", "Are all items relevant for the study population?", "Are all items relevant for the purpose of the application of the instrument?", and "Do all items together comprehensively reflect the construct to be measured?". To determine construct validity, Spearman rank correlation coefficients of the CCQ, MLHF-Q, and SF-36 were used, because most of the distributions were skewed. The SF-36 was used as the external criterion to assess construct validity. We predefined the hypotheses concerning the construct validity between corresponding domains: correlations $<0.30$ were considered as poor, correlations between $0.30-0.50$ as moderate, and $\geq 0.50$ as strong. ${ }^{22,23}$ Corresponding domains were defined as: CCQ total and MLHF-Q total score with all SF-36 domains. We expected the CCQ functional and MLHF-Q physical domains to correspond with the SF-36 domains physical functioning, social functioning, physical, and vitality; CCQ mental and MLHF-Q emotional domain with SF-36 domain mental health, social functioning, and role emotional; and CCQ symptom domain with SF-36 domain pain and vitality, and we found that these domains did correspond with each other. Correlations of $<0.1$ were expected for FEV 1 with MLHF-Q and CCQ total and domain scores. Construct validity was labeled as acceptable when $\geq 75 \%$ of the predefined hypotheses of the corresponding domains agreed. ${ }^{22}$ Internal consistency of the CCQ, MLHF-Q, and SF-36 was assessed using Cronbach's alpha coefficients; these were deemed adequate between 0.7 and $0.9 .{ }^{24}$ Reliability, test-retest reliability, and agreement were evaluated in stable patients after 2 weeks (defined here as GRC -1, 0, and 1). Test-retest reliability was assessed with intraclass correlation coefficients (ICCs) based on the two-way random effects model and the standard error of measurement (SEM), and test-retest reliability was assumed sufficient when ICC was $\geq 0.7 .{ }^{24}$ Agreement was assessed with a Bland-Altman plot for the CCQ and MLHF-Q total score. Agreement was defined as acceptable when the limits of agreement were smaller than the MCID. ${ }^{24-26}$

Responsiveness would be determined with the receiver operating characteristics (ROC) and the area under the curve (AUC), an adequate AUC was declared when at least 0.70 was declared as adequate. ${ }^{22,24}$ Floor and ceiling effects were assessed. When less than $15 \%$ of the patients achieved the highest or lowest possible score respectively, floor and ceiling effects were labeled absent and the test adequate..$^{22,25}$

\section{Results}

From October 25, 2011 to September 6, 2013, 58 patients were recruited and 50 completed the study, as shown in Figure 1. Patient demographic and clinical characteristics are presented in Table 1. All patients had COPD and HF; other common comorbidities were myocardial infarction $(31,53.4 \%)$, diabetes mellitus $(12,20.7 \%)$, and peripheral vascular disease $(17,29.3 \%)$.

\section{Validity \\ Face validity}

The CCQ and the MLHF-Q are both disease-specific questionnaires, separately designed for COPD and $\mathrm{HF}$, respectively. For use in patients with both morbidities, the items of both questionnaires have to reflect the construct "health status of patients with both COPD and HF". 


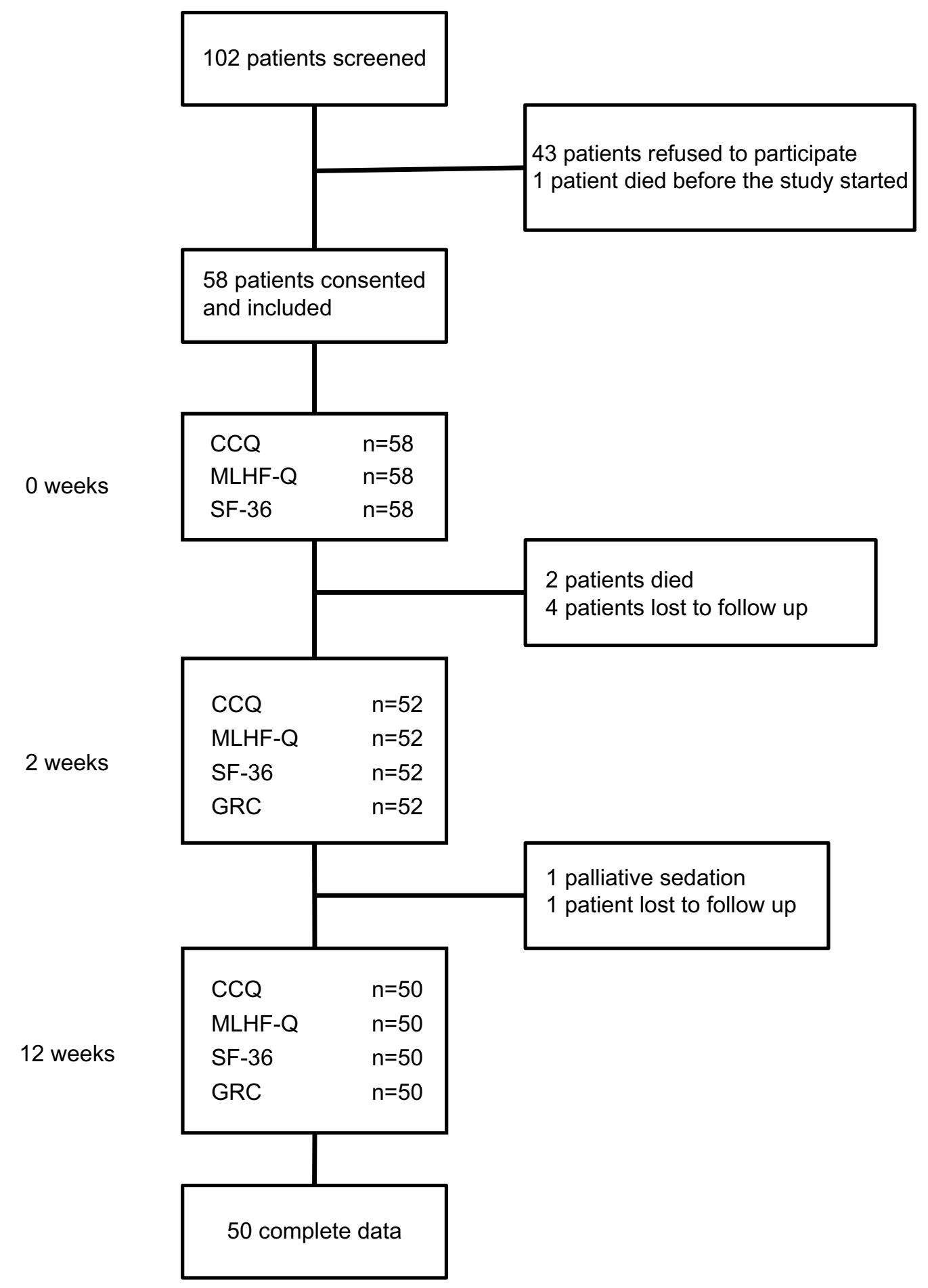

Figure I Flow chart showing the recruitment and retention of the study participants.

Note: In total 58 patients were included in the study, and 50 patients completed the study.

Abbreviations: CCQ, Clinical COPD Questionnaire; MLHF-Q, Minnesota for Living with Heart Failure Questionnaire; SF-36, Short-Form 36; GRC, Global Rating of Change.

Health status in patients with COPD and HF will partly derive from the severity of symptoms, ie, dyspnea, edema, orthopnea, cough, and phlegm. When the questionnaires are compared, the question "Did your HF prevent you from living as you wanted during the past month by causing swelling in your ankles or legs?" is missing in the CCQ, and the questions "How much of the time did you cough?" and "How much of the time did you produce phlegm?" were missing in the MLHF-Q. Except for the question about "ankle edema", all other questions of the MLHF-Q were comparable with the questions of the CCQ. 
Table I Patient characteristics

\begin{tabular}{|c|c|}
\hline$n$ & 58 \\
\hline Sex, male, n (\%) & $43(74.1)$ \\
\hline Age (years), mean (SD) & $73(6)$ \\
\hline Pack years, median (range) & $37.5(5-102)$ \\
\hline Current smoker, n (\%) & $17(29.3)$ \\
\hline Oxygen therapy, n (\%) & $11(19)$ \\
\hline BMI, mean (SD) & $27(5)$ \\
\hline $\begin{array}{l}\mathrm{FEV}_{\text {, (post-bronchodilator \% predicted), }} \\
\text { mean (SD) }\end{array}$ & $51(15)$ \\
\hline \multicolumn{2}{|l|}{ GOLD, n (\%) } \\
\hline 2 & $29(50)$ \\
\hline 3 & $16(27.6)$ \\
\hline 4 & $13(22.4)$ \\
\hline Pro-BNP (pg/mL), ${ }^{\text {a }}$ median (range) & $3,180(59-31,390)$ \\
\hline \multicolumn{2}{|l|}{ NYHA, n (\%) } \\
\hline 2 & $4 \mathrm{I}(70.7)$ \\
\hline 3 & $14(24.1)$ \\
\hline 4 & $3(5.2)$ \\
\hline \multicolumn{2}{|l|}{ Charlson Comorbidity Index, n (\%) } \\
\hline 2 & $12(20.7)$ \\
\hline 3 & $24(4 I .4)$ \\
\hline$\geq 4$ & $22(37.9)$ \\
\hline CCQ total score, mean (SD) & $2.7(I . I)$ \\
\hline MLHF-Q, mean (SD) & $43(22)$ \\
\hline SF-36, general health, mean (SD) & $31(18)$ \\
\hline
\end{tabular}

Notes: ${ }^{a} \mathrm{n}=57$. COPD classification by post-bronchodilator spirometry according to GOLD guidelines.

Abbreviations: $\mathrm{BMI}$, body mass index; $\mathrm{FEV}_{1}$, forced expiratory volume in one second; GOLD, Global Initiative for Chronic Obstructive Lung Disease; proBNP, pro-B-type natriuretic peptide; NYHA, New York Heart Association; CCQ, Clinical COPD Questionnaire; MLHF-Q, Minnesota for Living with Heart Failure Questionnaire; SF-36, Short-Form 36; SD, standard deviation.

\section{Content validity}

1. "Do all items of the CCQ and MLHF-Q refer to relevant aspects of health status in patients with COPD and HF?" Originally, the CCQ was developed and validated in COPD patients, ${ }^{12}$ and the MLHF-Q was developed and validated in patients with HF. ${ }^{13}$ Since symptoms in COPD patients and in HF patients show considerable overlap, most of the items in both questionnaires reflect health status for both diseases, ie, COPD and HF.

2. "Are all items relevant for patients with COPD and HF?" The CCQ lacks an item about ankle edema, ${ }^{12}$ and the MLHF-Q lacks items about cough and phlegm. ${ }^{13}$ All other items are similar for both questionnaires.

3. "Are all items relevant for the purpose of application of the CCQ and MLHF-Q?" Ideally, a disease-specific questionnaire for patients with both COPD and HF should be developed, and should have good discriminative and evaluative properties. However, both questionnaires ${ }^{12,13}$ are largely equal, and theoretically the authors consider therefore that both questionnaires perhaps can be used in patients with COPD and HF.
4. "Do all items together comprehensively reflect health status in patients with COPD and HF?" Based on previous validation studies, ${ }^{12,27,28}$ the CCQ reflects health status in COPD patients but is also validated in other patient populations, including patients with laryngotracheal stenosis. The MLHF-Q reflects health status in HF patients, ${ }^{13}$ patients with atrial fibrillation, ${ }^{29}$ and patients with heart valve surgery. ${ }^{30}$ Comprehensive evaluation of health status in patients with both COPD and HF has not been assessed yet for either questionnaire.

\section{Construct validity}

The correlation coefficients between the corresponding domains of the CCQ and the MLHF-Q with the external criterion, the SF-36, are shown in Table 2. Most of the corresponding domains between the SF-36 and the CCQ and MLHF-Q show moderate to strong correlations, except for the SF-36 role emotional domain and the corresponding MLHF-Q emotional domain $(-0.27)$ and the SF-36 role physical and the corresponding MLHF-Q physical domain $(-0.29)$. Convergent validity is depicted in Table 3. As hypothesized, all corresponding domains of the CCQ and the MLHF-Q had strong correlations $(\geq 0.50)$. Conversely, correlations between the CCQ and MLHF-Q questionnaires and $\mathrm{FEV}_{1}$ percent predicted were indeed low, although some did slightly surpass the 0.1 boundary (Table 3 ). The predefined hypothesis for the corresponding domains of the CCQ, MLHF-Q, SF-36, and FEV 1 percent predicted agreed in $75 \%$ of cases.

\section{Reliability Internal consistency}

All Cronbach's alpha values were $>0.7$, implying satisfactory internal consistency for the CCQ and MLHF-Q total and domain scores and most of the SF-36 domain scores, except for the domains general health (0.63) and social $(0.69$, see Table 4$)$.

\section{Test-retest reliability}

The ICC was tested in 33 patients who remained stable after 2 weeks and was adequate $(\geq 0.7)$ for all questionnaires, ie, the CCQ, MLHF-Q, and SF-36, indicating good test-retest reliability (Table 5 ). The only exceptions that had lower ICCs were: the CCQ symptom score $(0.42$ $[0.11,0.66])$, SF-36 physical functioning $(0.64[0.32$, $0.82])$, and SF-36 role emotional $(0.24[-0.13,0.53])$. The SEMs of the total and domain scores of all questionnaires were larger than the MCID on the individual 
Table 2 Construct validity $(n=58)$

\begin{tabular}{|c|c|c|c|c|}
\hline & \multicolumn{4}{|l|}{ CCQ } \\
\hline & Total & Functional & Mental & Symptom \\
\hline \multicolumn{5}{|l|}{ SF-36 } \\
\hline General health & $-0.57(<0.00 \mathrm{I})$ & $-0.53(<0.00 \mathrm{I})$ & $-0.30(0.02)$ & $-0.5 \mathrm{I}(<0.00 \mathrm{I})$ \\
\hline Role physical & $-0.32(0.01)$ & $-0.25(0.06)$ & $-0.2 \mathrm{I}(0 . \mathrm{II})$ & $-0.26(0.05)$ \\
\hline Pain & $-0.44(0.001)$ & $-0.36(0.006)$ & $-0.36(0.006)$ & $-0.31(0.02)$ \\
\hline Physical functioning & $-0.63(<0.001)$ & $-0.77(<0.00 \mathrm{I})$ & $-0.44(0.00 \mathrm{I})$ & $-0.34(0.008)$ \\
\hline Vitality & $-0.65(<0.001)$ & $-0.50(<0.001)$ & $-0.60(<0.001)$ & $-0.53(<0.001)$ \\
\hline Social functioning & $-0.62(<0.001)$ & $-0.60(<0.001)$ & $-0.59(<0.001)$ & $-0.38(0.003)$ \\
\hline Role emotional & $-0.30(0.02)$ & $-0.23(0.09)$ & $-0.33(0.01)$ & $-0.22(0.10)$ \\
\hline \multirow[t]{3}{*}{ Mental health } & $-0.50(<0.00 \mathrm{I})$ & $-0.27(0.04)$ & $-0.62(<0.00 \mathrm{I})$ & $-0.38(0.004)$ \\
\hline & \multicolumn{4}{|c|}{ MLHF-Q $(n=58)$} \\
\hline & Total & Physical & Emotional & \\
\hline \multicolumn{5}{|l|}{ SF-36 } \\
\hline General health & $-0.50(<0.00 \mathrm{I})$ & $-0.48(<0.00 \mathrm{I})$ & $-0.38(0.004)$ & \\
\hline Role physical & $-0.37(0.005)$ & $-0.29(0.03)$ & $-0.34(0.008)$ & \\
\hline Pain & $-0.40(0.002)$ & $-0.37(0.004)$ & $-0.36(0.006)$ & \\
\hline Physical functioning & $-0.5 \mathrm{I}(<0.00 \mathrm{I})$ & $-0.63(<0.001)$ & $-0.29(0.03)$ & \\
\hline Vitality & $-0.64(<0.001)$ & $-0.6 \mathrm{I}(<0.00 \mathrm{I})$ & $-0.53(<0.001)$ & \\
\hline Social functioning & $-0.6 \mathrm{I}(<0.00 \mathrm{I})$ & $-0.62(<0.001)$ & $-0.60(<0.001)$ & \\
\hline Role emotional & $-0.33(0.01)$ & $-0.27(0.04)$ & $-0.27(0.05)$ & \\
\hline Mental health & $-0.53(<0.001)$ & $-0.46(<0.001)$ & $-0.60(<0.001)$ & \\
\hline
\end{tabular}

Notes: Construct validity is presented with Spearman rank correlations ( $P$-value). Correlations of the corresponding domains of $\geq 0.5$ are adequate according to the hypothesis described in the statistical section.

Abbreviations: CCQ, Clinical COPD Questionnaire; SF-36, Short Form 36; MLHF-Q, Minnesota for Living with Heart Failure Questionnaire.

level, except for the MLHF-Q emotional domain (SEM 2.02).

\section{Agreement}

Bland-Altman plots of the CCQ total score and MLHF-Q total score for the 33 patients who remained stable after 2 weeks are shown in Figure 2. The mean difference after 2 weeks was $-0.26 \pm 0.14$ for the CCQ total score and $-3.64 \pm 2.15$ for the MLHF-Q total score. The upper and lower limits of agreement were 1.35 and -1.87 for the CCQ total score. The MLHF-Q had an upper limit of agreement of 20.52 and a lower limit of agreement of -27.80 .

\section{Measurement of change} Responsiveness

Patients in this study were in a stable phase of their disease, ie, $\mathrm{HF}$ and COPD. After inclusion, patients received no change in intervention and were not expected to improve much. Therefore, responsiveness could not be assessed in this study.

\section{Interpretability}

Floor effects (lowest score) were present in the CCQ mental domain 36.2\% and in the MLHF-Q emotional domain $20.7 \%$. The SF-36 showed floor effects for the domains role

Table 3 Construct validity: convergent and divergent validity $(n=58)$

\begin{tabular}{|c|c|c|c|c|}
\hline Convergent validitya & MLHF-Q total & MLHF-Q physical & MLHF-Q emotional & \\
\hline $\mathrm{CCQ}$ total & $0.84(<0.001)$ & $0.82(<0.00 \mathrm{I})$ & $0.57(<0.00 \mathrm{I})$ & \\
\hline CCQ symptom & $0.6 \mathrm{I}(<0.00 \mathrm{I})$ & $0.54(<0.001)$ & $0.43(<0.001)$ & \\
\hline CCQ functional & $0.67(<0.001)$ & $0.72(<0.001)$ & $0.35(0.007)$ & \\
\hline CCQ mental & $0.69(<0.001)$ & $0.68(<0.001)$ & $0.65(<0.001)$ & \\
\hline Divergent validity & CCQ total & CCQ symptom & CCQ functional & CCQ mental \\
\hline \multirow[t]{2}{*}{$\mathrm{FEV}, \%$ predicted } & $-0.17(0.20)$ & $-0.16(0.23)$ & $-0.17(0.20)$ & $0.03(0.85)$ \\
\hline & MLHF-Q total & MLHF-Q physical & MLHF-Q emotional & \\
\hline $\mathrm{FEV}, \%$ predicted & $0.04(0.76)$ & $0.04(0.74)$ & $0.10(0.46)$ & \\
\hline
\end{tabular}

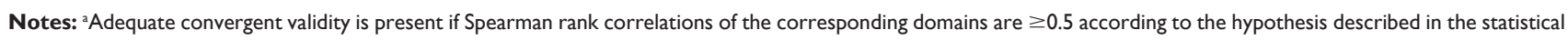
section; 'Spearman rank correlations of divergent validity were expected to be $\leq 0$. I.

Abbreviations: CCQ, Clinical COPD Questionnaire; MLHF-Q, Minnesota for Living with Heart Failure Questionnaire; FEV , forced expiratory volume in one second. 
Table 4 Internal consistency $(n=58)$

\begin{tabular}{ll}
\hline & Cronbach's $\alpha$ \\
\hline CCQ & \\
Total & 0.87 \\
Mental & 0.80 \\
Symptom & 0.75 \\
Functional & 0.86 \\
MLHF-Q & \\
Total & 0.91 \\
Emotional & 0.90 \\
Physical & 0.86 \\
SF-36 & \\
General health & 0.63 \\
Mental health & 0.83 \\
Role emotional & 0.86 \\
Role physical & 0.91 \\
Physical functioning & 0.88 \\
Social functioning & 0.69 \\
Vitality & 0.80 \\
Pain & 0.92 \\
\hline
\end{tabular}

Notes: Internal consistency is assessed with the Cronbach's $\alpha$ coefficient, a correlation between 0.70 and 0.95 is considered as good internal consistency.

Abbreviations: CCQ, Clinical COPD Questionnaire; MLHF-Q, Minnesota for Living with Heart Failure Questionnaire; SF-36, Short Form 36.

emotional (25.9\%) and physical functioning (75.9\%) and ceiling effects (highest score) for the domains role emotional $(50 \%)$ and pain $(36.2 \%)$, as shown in Table 6.

\section{Discussion}

This is the first study comparing and validating the CCQ and MLHF-Q in patients with coexisting COPD and HF.

Table 5 Test-retest reliability $(n=33)$

\begin{tabular}{lll}
\hline & ICC $(95 \% \mathbf{C l})$ & SEM \\
\hline CCQ & & \\
Total & $0.70(0.48,0.84)$ & 0.60 \\
Mental & $0.75(0.55,0.87)$ & 0.67 \\
Symptom & $0.42(0.10,0.66)$ & 0.89 \\
Functional & $0.79(0.63,0.89)$ & 0.69 \\
MLHF-Q & & \\
Total & $0.85(0.71,0.92)$ & 8.96 \\
Emotional & $0.90(0.80,0.95)$ & 2.02 \\
Physical & $0.79(0.62,0.89)$ & 5.17 \\
SF-36 & & \\
General health & $0.74(0.54,0.86)$ & 9.35 \\
Mental health & $0.79(0.61,0.89)$ & 8.74 \\
Role emotional & $0.24(-0.13,0.65)$ & 36.00 \\
Role physical & $0.90(0.80,0.95)$ & 8.70 \\
Physical functioning & $0.64(0.32,0.82)$ & 25.75 \\
Social functioning & $0.80(0.64,0.90)$ & 12.12 \\
Vitality & $0.76(0.57,0.88)$ & 11.54 \\
Pain & $0.82(0.67,0.91)$ & 12.19 \\
\hline Notes Tst-retstra &
\end{tabular}

Notes: Test-retest-reliability is presented with the ICC and $95 \% \mathrm{Cl}$. ICC $\geq 0.70$ gives a positive rating for test-retest reliability. Only stable patients (Global Rating of Change $-\mathrm{I}, 0, \mathrm{I}$ ).

Abbreviations: CCQ, Clinical COPD Questionnaire; MLHF-Q, Minnesota for Living with Heart Failure Questionnaire; SF-36, Short-Form 36; SEM, standard error of measurement; ICC, intraclass correlation coefficient; $\mathrm{Cl}$, confidence interval.
The discriminative part of the psychometric properties, ie, validity, internal consistency, and test-retest reliability, were comparable for the CCQ and MLHF-Q. The only exception was the CCQ symptom score, in which weaker correlations were found for test-retest reliability and construct validity. The evaluative part, ie, agreement of the CCQ and MLHF-Q, was similarly limited in both. The psychometric properties of both questionnaires will be discussed separately. Because the CCQ and MLHF-Q have not been validated before in patients with both COPD and HF, we cannot compare our findings directly with similar validation studies. Therefore, to give some perspective and to quantify our findings, we compared our study with validation studies in other patient populations.

Construct validity was assessed between the external criterion, ie, the SF-36, and both questionnaires, ie, the CCQ and MLHF-Q. Most of the correlations between the corresponding domains of the SF-36 and the MLHF-Q were moderate to strong. Other validation studies ${ }^{29,30}$ in patients with atrial fibrillation and patients undergoing heart valve surgery found similar correlations between the SF-36 and MLHF-Q. Corresponding domains of the CCQ and SF-36 achieved moderate to strong correlations as well, and were comparable with most of the correlations found in the original validation study. ${ }^{12}$ The exception to this notion is the CCQ symptom domain, which seems to have lower correlations with SF-36 in comparison with the original validation study in patients with COPD only. ${ }^{12}$ This might signify that the CCQ symptom domain is not a reflection of the most important symptoms of patients with combined COPD and HF. The symptoms in COPD and HF overlap partially; however, some symptoms are different, like orthopnea or edema. ${ }^{31}$ The CCQ symptom domain consists of four items: "Short of breath at rest?", "Short of breath doing physical activities?", "Did you cough?", and "Did you produce phlegm?". These questions reflect symptom-related health status for patients with COPD alone. Symptom-related health status for patients with both diseases, ie, COPD and HF, is probably more complex.

The test-retest reliability of the CCQ symptom domain was also limited, with an ICC of $0.42(0.10,0.66)$. Previous studies in other populations showed higher ICCs for the CCQ symptom domain; one study ${ }^{27}$ in patients with COPD in primary care found an ICC of 0.74 and another study ${ }^{28}$ in adults with laryngotracheal stenosis found an ICC of 0.94 . We do not have an explanation for this, but have considered whether the low ICC in our study could be due to the fact that patients with both COPD and HF are a more homogeneous population than expected. In that case, the variability of 


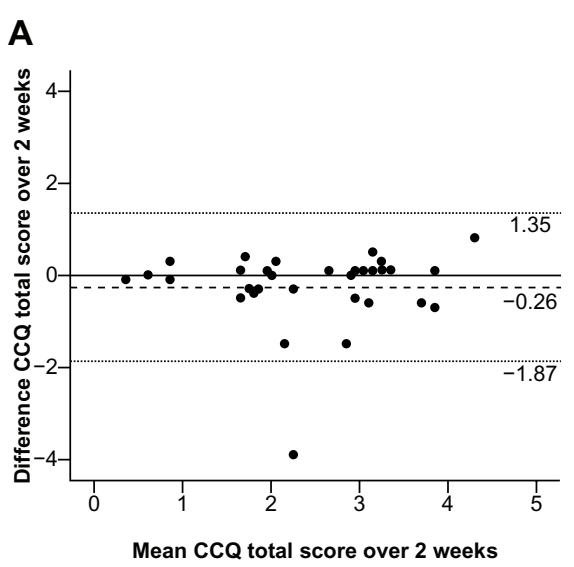

\section{B}

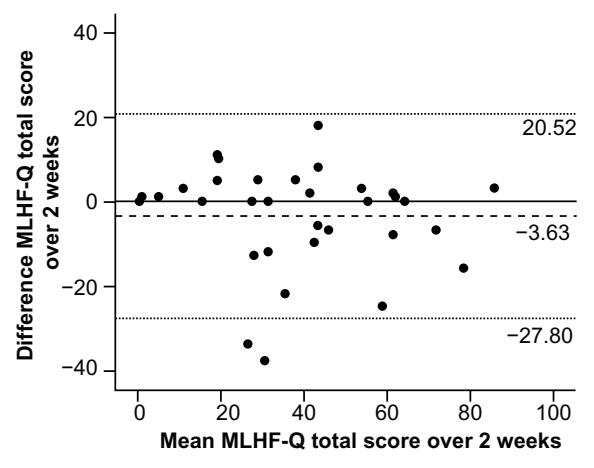

Figure 2 Agreement over time of CCQ total score and MLHF-Q total score in stable COPD patients. (A) Agreement of the CCQ total score and (B) agreement of the MLHF-Q total score over 2 weeks in stable COPD patients (GRC - I, 0, I).

Notes: The bold flat line represents the mean difference over 2 weeks for the CCQ and MLHF-Q total scores. The dashed lines are the limits of agreement, I.96× standard deviation. ( $n=33)$.

Abbreviations: COPD, chronic obstructive pulmonary disease; CCQ, Clinical COPD Questionnaire; MLHF-Q, Minnesota for Living with Heart Failure Questionnaire; GRC, Global Rating of Change.

scores on the CCQ symptom domain between patients is low and within patients are equally present, leading to a low ICC and hampering its use as a discriminative tool in patients with both COPD and HF.

The MLHF-Q physical domain consists of eight items, with six items containing questions about functional state and two items canvassing symptom-related health status, ie, "Did your heart failure prevent you from living as you wanted during the last month by making you short of breath?", and "Did your heart failure prevent you from living as you wanted during the last month by making you tired, fatigued, or low on energy?". ${ }^{13}$ The MLHF-Q physical domain has an adequate

Table 6 Floor and ceiling effect $(n=58)$

\begin{tabular}{llll}
\hline & Mean (SD) & Floor (\%) & Ceiling (\%) \\
\hline CCQ & & & \\
Total & $2.7(I . I)$ & 1.7 & 0 \\
Mental & $1.3(1.4)$ & 36.2 & 0 \\
Symptom & $3.0(1.3)$ & 1.7 & 1.7 \\
Functional & $3.2(1.4)$ & 1.7 & 0 \\
MLHF-Q & & & \\
Total & $43.3(22.5)$ & 5.2 & 0 \\
Emotional & $7.2(6.5)$ & 20.7 & 0 \\
Physical & $22.3(10.9)$ & 5.2 & 0 \\
SF-36 & & & \\
General health & $31(18.1)$ & 3.4 & 0 \\
Mental health & $72.4(19.0)$ & 0 & 5.2 \\
Role emotional & $60.3(43.5)$ & 25.9 & 50 \\
Role physical & $32.1(25.2)$ & 12.1 & 0 \\
Physical functioning & $15.1(31.0)$ & 75.9 & 8.6 \\
Social functioning & $60.3(27.4)$ & 3.4 & 19 \\
Vitality & $44.7(31.0)$ & 0 & 0 \\
Pain & $66.2(32.0)$ & 1.7 & 36.2 \\
\hline Abrevations: &
\end{tabular}

Abbreviations: CCQ, Clinical COPD Questionnaire; MLHF-Q, Minnesota for Living with Heart Failure Questionnaire; SF-36, Short-Form 36; SD, standard deviation. discriminative value in patients with COPD and HF, with an ICC of $0.79(0.62,0.89)$. Perhaps this is due to the combination of functional state and symptoms-related questions about health status in the MLHF-Q physical domain. The discriminative value in patients with COPD and HF is adequate as well, with an ICC of $0.79(0.63,0.89)$.

The degree of interrelatedness between the items of the domains of the CCQ and the MLHF-Q, internal consistency, were adequate, all Cronbach's alpha coefficients were $\geq 0.70$, and were comparable with previous validation studies $^{12,13,30}$ in patients with COPD, heart valve surgery, or HF.

The SEM of the CCQ and MLHF-Q total and domain scores on the individual level was larger than the MCID of both questionnaires. This suggests that neither questionnaire could differentiate between a clinical relevant change and measurement error in our study. Two studies have addressed the SEM of the CCQ total score before and found SEMs of $0.29^{32}$ and $0.21 .^{33}$ However, these SEMs were determined in COPD patients without HF. In our study, an SEM of 0.60 was found for the CCQ total score. The standard measurement error can be calculated on group level and on individual level (per patient). The SEM on individual level can be interpreted and used in clinical practice in the outpatient clinic. The SEM on group level can be interpreted and used in groups, ie clinical research. The SEMs on the group level for the CCQ total, symptom, functional, and mental scores were $0.10,0.15,0.12$, and 0.11 , respectively. The latter SEM values are all smaller than the MCID of the CCQ, 0.4 points. Thus, on the group level, the CCQ is able to differentiate a real change from measurement error. The SEMs for the MLHF-Q total, emotional, and physical score on the group level were $1.56,0.35$, and 0.90 , respectively. 
The MCID of the MLHF-Q is 4.8. Also, the MLHF-Q was able to differentiate clinically relevant change from measurement error in patients with COPD and HF on the group level.

Similar results were found for the agreement. Both questionnaires had large limits of agreement on the individual level, ie, 1.35 and -1.87 for the CCQ total score and 20.52 and -27.80 for the MLHF-Q total score. On the group level, the limits of agreement were 0.23 and -0.32 for the CCQ total score and 3.5 and -4.84 for the MLHF-Q. On the group level, the limits of agreement are smaller or comparable with the MCID for both questionnaires, indicating that a clinically relevant change in health status can be distinguished from measurement error.

This study has some limitations that are worth discussing. One limitation is that the responsiveness, ie, the ability of the CCQ and MLHF-Q to detect changes in health status over time, could not be assessed in this study because patients received no change in intervention. Therefore, no conclusion can be drawn regarding patients who improved or deteriorated versus those who remained stable. Most of the patients remained stable because no intervention was given. Another limitation is the use of the SF-36, a generic health status questionnaire, as the reference standard; ideally, a disease-specific health status questionnaire for patients with COPD and HF would be used. Unfortunately, there is no such questionnaire for these patients. We chose generic SF-36 because it is validated for both COPD and HF. ${ }^{34,35}$ The last limitation is the MCIDs used for the CCQ and MLHFQ. These MCIDs were determined for patients with either COPD or HF, but not in patients with combined disease. This could underestimate or overestimate interpretation of the agreement, because agreement was acceptable when the limits of agreement were smaller than the MCID.

In conclusion, both questionnaires, the CCQ and the MLHF-Q, are valid and reliable for patients with both COPD and HF on the group level, for instance in clinical research or validation studies. However, the CCQ symptom domain does not reflect all symptoms of patients with coexistent COPD and HF, limiting its usefulness in this setting. On the individual level, ie, in clinical practice, the CCQ and MLHF-Q were not able to differentiate a real clinically relevant change from measurement error in patients with COPD and HF in this study. Ideally, a new questionnaire should be developed, whereby a more complete reflection of health status can be measured in patients with both COPD and HF.

\section{Acknowledgments}

We would like to acknowledge Stichting Astma Bestrijding for a subvention in support of this work. We also thank the Isala Hospital for a health innovation science grant.

\section{Disclosure}

The authors report no conflicts of interest in this work.

\section{References}

1. Sansores RH, Ramirez-Venegas A, Hernandez-Zenteno R, MayarMaya ME, Perez-Bautista OG, Velazquez UM. Prevalence and diagnosis of chronic obstructive pulmonary disease among smokers at risk. A comparative study of case-finding vs screening strategies. Respir Med. 2013;107:580-586.

2. Fabbri LM, Luppi F, Beghe B, Rabe KF. Complex chronic comorbidities of COPD. Eur Respir J. 2008;31:204-212.

3. Hawkins NM, Petrie MC, Jhund PS, Chalmers GW, Dunn FG, McMurray JJ. Heart failure and chronic obstructive pulmonary disease: diagnostic pitfalls and epidemiology. Eur J Heart Fail. 2009;11:130-139.

4. Sin DD, Man SF. Why are patients with chronic obstructive pulmonary disease at increased risk of cardiovascular diseases? The potential role of systemic inflammation in chronic obstructive pulmonary disease. Circulation. 2003;107:1514-1519.

5. Mentz RJ, Fiuzat M, Wojdyla DM, et al. Clinical characteristics and outcomes of hospitalized heart failure patients with systolic dysfunction and chronic obstructive pulmonary disease: findings from OPTIMIZE-HF. Eur J Heart Fail. 2012;14:395-403.

6. Brenner S, Guder G, Berliner D, et al. Airway obstruction in systolic heart failure - COPD or congestion? Int J Cardiol. 2013;168: 1910-1916.

7. Lee $\mathrm{D}$, Wingate $\mathrm{S}$. Managing the heart failure patient with obstructive lung disease. J Cardiovasc Nurs. 2004;19:S27-S34.

8. Almagro P, Cabrera FJ, Diez J, et al. Comorbidities and short-term prognosis in patients hospitalized for acute exacerbation of COPD The ESMI study. Chest. 2012;141:851-857.

9. Seemungal TA, Donaldson GC, Paul EA, Bestall JC, Jeffries DJ, Wedzicha JA. Effect of exacerbation on quality of life in patients with chronic obstructive pulmonary disease. Am J Respir Crit Care Med. 1998;157:1418-1422.

10. Patel AR, Donaldson GC, Mackay AJ, Wedzicha JA, Hurst JR. The impact of ischemic heart disease on symptoms, health status, and exacerbations in patients with COPD. Chest. 2012;141:851-857.

11. Vestbo J, Hurd SS, Agusti AG, et al. Global strategy for the diagnosis, management, and prevention of chronic obstructive pulmonary disease: GOLD executive summary. Am J Respir Crit Care Med. 2013; 187:347-365.

12. van der Molen T, Willemse BW, Schokker S, ten Hacken NH, Postma DS, Juniper EF. Development, validity and responsiveness of the Clinical COPD Questionnaire. Health Qual Life Outcomes. $2003 ; 1: 13$.

13. Rector TS, Kubo SH, Cohn JN. Assessment of patients' self-assessment of their heart failure: content, reliability and validity of a new measureThe Minnesota Living with Heart Failure Questionnaire. Heart Fail. 1987;3:198-209.

14. McKelvie RS, Moe GW, Ezekowitz JA, et al. The 2012 Canadian Cardiovascular Society heart failure management guidelines update: focus on acute and chronic heart failure. Can J Cardiol. 2013;29: $168-181$.

15. Kocks JW, Tuinenga MG, Uil SM, van den Berg JW, Stahl E, van der MT. Health status measurement in COPD: the minimal clinically important difference of the clinical COPD questionnaire. Respir Res. 2006;7:62.

16. Bennett SJ, Oldridge NB, Eckert GJ, et al. Comparison of quality of life measures in heart failure. Nurs Res. 2003;52:207-216.

17. McHorney CA, Ware JE Jr, Raczek AE. The MOS 36-Item ShortForm Health Survey (SF-36): II. Psychometric and clinical tests of validity in measuring physical and mental health constructs. Med Care. 1993;31:247-263.

18. Ware JE Jr, Sherbourne CD. The MOS 36-item Short-Form health survey (SF-36). I. Conceptual framework and item selection. Med Care. 1992;30:473-483. 
19. Aaronson NK, Muller M, Cohen PD, et al. Translation, validation, and norming of the Dutch language version of the SF-36 Health Survey in community and chronic disease populations. J Clin Epidemiol. 1998;51: 1055-1068.

20. Kamper S. Global Rating of Change scales. Aust J Physiother. 2009;55:289.

21. Mokkink LB, Terwee CB, Patrick DL, et al. The COSMIN study reached international consensus on taxonomy, terminology, and definitions of measurement properties for health-related patient-reported outcomes. J Clin Epidemiol. 2010;63:737-745.

22. de Vet HC, Terwee CB, Mokkink LB, Knol DL. Practical guides to biostatic and epirdemiology. In: Measurement in Medicine: A Practical Guide. Cambridge Books Online: Cambridge University Press; 2013. Available from: ebooks.cambridge.org/ebook. jsf?bid=CBO9780511996214. Accessed June 19, 2014.

23. Agusti A, Soler JJ, Molina J, et al. Is the CAT questionnaire sensitive to changes in health status in patients with severe COPD exacerbations? COPD. 2012;9:492-498.

24. Terwee CB, Bot SD, de Boer MR, et al. Quality criteria were proposed for measurement properties of health status questionnaires. J Clin Epidemiol. 2007;60:34-42.

25. Streiner DL, Norman GR. Health Measurement Scales. A Practical Guide to Their Development Uses. 4th ed. Oxford, UK: Oxford University Press; 2008. Available from: http://global.oup.com/academic/ product/health-measurement-scales-9780199231881; jsessionid=46EA BF91F5A596CFCED05CD43DB5A31B?cc=nz\&lang=en\&. Accessed June 19, 2014.

26. Bland JM, Altman DG. Measuring agreement in method comparison studies. Stat Methods Med Res. 1999;8:135-160.
27. Stallberg B, Nokela M, Ehrs PO, Hjemdal P, Jonsson EW. Validation of the clinical COPD Questionnaire (CCQ) in primary care. Health Qual Life Outcomes. 2009;7:26.

28. Nouraei SA, Randhawa PS, Koury EF, et al. Validation of the Clinical COPD Questionnaire as a psychophysical outcome measure in adult laryngotracheal stenosis. Clin Otolaryngol. 2009;34:343-348.

29. Middel B, Bouma J, de Jongste M, et al. Psychometric properties of the Minnesota Living with Heart Failure Questionnaire (MLHF-Q). Clin Rehabil. 2001;15:489-500.

30. Supino PG, Borer JS, Franciosa JA, et al. Acceptability and psychometric properties of the Minnesota Living With Heart Failure Questionnaire among patients undergoing heart valve surgery: validation and comparison with SF-36. J Card Fail. 2009;15:267-277.

31. Hannink JD, van Helvoort HA, Dekhuijzen PN, Heijdra YF. Heart failure and COPD: partners in crime? Respirology. 2010;15: 895-901.

32. Kon SS, Dilaver D, Mittal M, et al. The Clinical COPD Questionnaire: response to pulmonary rehabilitation and minimal clinically important difference. Thorax. October 22, 2013. [Epub ahead of print.]

33. Tsiligianni IG, van der Molen T, Moraitaki D, et al. Assessing health status in COPD. A head-to-head comparison between the COPD assessment test (CAT) and the clinical COPD questionnaire (CCQ). BMC Pulm Med. 2012;12:20.

34. Mahler DA, Mackowiak JI. Evaluation of the Short-Form 36-item questionnaire to measure health-related quality of life in patients with COPD. Chest. 1995;107:1585-1589.

35. Saccomann IC, Cintra FA, Gallani MC. Health-related quality of life among the elderly with heart failure: a generic measurement. Sao Paulo Med J. 2010;128:192-196.
International Journal of COPD

\section{Publish your work in this journal}

The International Journal of COPD is an international, peer-reviewed journal of therapeutics and pharmacology focusing on concise rapid reporting of clinical studies and reviews in COPD. Special focus is given to the pathophysiological processes underlying the disease, intervention programs, patient focused education, and self management protocols.

\section{Dovepress}

This journal is indexed on PubMed Central, MedLine and CAS. The manuscript management system is completely online and includes a very quick and fair peer-review system, which is all easy to use. Visit $\mathrm{http}: / /$ www.dovepress.com/testimonials.php to read real quotes from published authors. 